\title{
Plug-In Hybrid Electric Vehicles in Dynamical Energy Markets
}

\author{
J.T.B.A. Kessels \\ TNO Automotive, \\ P.O. Box 756, 5700 AT Helmond, \\ The Netherlands \\ john.kessels@tno.nl
}

\author{
P.P.J. van den Bosch \\ Technische Universiteit Eindhoven, \\ Dept. of Electrical Engineering, \\ P.O. Box 513, 5600 MB Eindhoven, \\ The Netherlands \\ $p \cdot p \cdot j \cdot v \cdot d \cdot b o s c h a t u e \cdot n l$
}

\begin{abstract}
The plug-in hybrid electric vehicle allows vehicle propulsion from multiple internal power sources. Electric energy from the grid can be utilized by means of the plug-in connection. An on-line energy management (EM) strategy is proposed to minimize the costs for taking energy from each power source. Especially in a dynamical energy market, an on-line optimization algorithm is desirable since energy prices change over time.

By construction, the proposed EM system can operate with, and without prediction information. If predictions are available, an electronic horizon is applied to anticipate on up-coming events and further optimize the strategy. Illustrative examples are given to explain the added value for both solutions. Also the situation where energy is transferred back to the grid is considered.
\end{abstract}

\section{INTRODUCTION}

Today's road traffic plays an important part in the energy consumption world wide. The traditional energy sources are running out and the world has to focuss on sustainable and environmentally friendly solutions for transportation. By enabling a free energy market, governments stimulate a technology-push for novel solutions where supply and demand can become price-sensitive and react to the varying prices for energy.

In line with this reasoning, this paper proposes an intelligent energy management system for plug-in hybrid electric vehicles. The plug-in hybrid electric vehicle (PI-HEV) is characterized as follows. The vehicle entails at least two power sources, which can both supply power for vehicle propulsion. This is similar to a hybrid electric vehicle (HEV), where typically an internal combustion engine (ICE) is the primary power source (powered by fuel in the tank) and the secondary power source is an electric machine connected to the battery. Except for these internal power sources, the PI-HEV can also benefit from external power sources. Therefore, it is equipped with a plug-in cable to be connected to the grid.

Due to its sophisticated power net topology, a PI-HEV needs to be equipped with an energy management (EM) strategy. At present, many well-defined solutions have been presented for HEVs, usually aiming at maximizing the vehicle energy efficiency. An excellent overview can be found in [1]. Typically, HEVs keep the energy content of the battery in a restricted operating window, to ensure a charge sustaining strategy for the battery. This artificial constraint hampers the economic benefits from the secondary power source, although the battery life time is extended since its energy throughput remains limited. In contrast, a full electric vehicle (EV) deals with a charge depleting strategy. This means that the batteries will be discharged during driving and after the trip, they need to be recharged, usually from the grid. It is obvious that the driving range of an EV is limited by its storage capacity of the battery. Moreover, it is far from trivial to estimate the actual status of the battery and the available storage capacity, so safety measures will be needed to prevent early battery depletion. Typically, safety margins are introduced which confine the true storage capacity of the battery to increase vehicle reliability. More details about hybrid and electric vehicles can be found in [2].

In the field of PI-HEVs, most work concentrates on the conversion of a series-production HEV into a PI-HEV, see e.g. [3], [4]. Typically, an upgrade of the battery is done for extra storage capacity and an energy plug with an AC/DC converter is installed to charge the battery from the grid. Nevertheless, only minor changes are done to the vehicle software, so automatically a charge-depleting EM strategy emerges when the battery has been charged from the grid. As soon as the battery enters its nominal operating range, the EM system returns to its normal charge sustaining strategy (similar to HEVs) and the battery is not further depleted, see [5]. It is clear that a PI-HEV deserves a more advanced EM strategy to benefit from the extra connection with the grid.

Especially in a dynamical energy market, it is important to decide when it is economically attractive to charge the vehicle from the grid. Whereas some strategies always decide to plug-in the vehicle and charge the batteries [7], it is already recognized that energy prices might be too high and it is economically attractive to propel the vehicle by its primary power source only [8]. Unfortunately, many of these advanced EM strategies suffer from a large computational demand since they rely on complex optimization algorithms. To obtain a system which can be implemented on-line in the vehicle, a sub-optimal rule-based solution is frequently proposed [7].

This paper presents an EM system which is directly suitable for on-line implementation. The concept originates from the fuel equivalent minimization strategy, which readily becomes a defacto standard for EM in HEVs [1]. Two separate modes are defined to discriminate between driving situations 
and moments when the vehicle is connected to the grid. By considering a physical interpretation for the decisions taken by the EM strategy, the computational demand reduces to a comparison between the cost for producing electric energy with the vehicle and the costs to buy electricity from the grid. Since the energy prices are on-line updated, the strategy automatically adapts to changes in the energy market.

It is also observed that knowledge about the future driving cycle, including the costs to buy electricity from the grid, offers interesting challenges to further optimize the profits from the EM system. By extending the EM strategy with an electronic horizon (e-horizon), it is demonstrated how to anticipate on upcoming events and maximize the economic benefits of the PI-HEV. Furthermore, this paper also considers the situation where the PI-HEV operates in a stationary power take-off situation, where it delivers power back to the grid. In future, this can be an important feature to guarantee stability of the grid in case more (renewable) energy sources will be connected. Nevertheless, a bi-directional AC/DC converter has to be installed in the vehicle to exchange energy between the grid and the battery in both directions. Also component sizing falls outside the scope of this paper. It is assumed that an appropriate vehicle configuration has been selected for the PI-HEV, including a suitable AC/DC converter. For a general overview of hardware requirements for the PI-HEV, the interested reader is referred to [5], [6].

This paper is build up as follows. Section II briefly describes the fuel equivalent minimization strategy and explains how a PI-HEV will benefit from this concept. Next, a formal description of the EM strategy is given in Section III, including directions for on-line implementation. Some illustrative examples about the cost benefits of this strategy are discussed in Section IV. The e-horizon extends the EM system with prediction information in Section V. Guidelines about the implementation are described in this section as well. Finally, concluding remarks are given in Section VI.

\section{FUEL EQUIVALENT MINIMIZATION STRATEGY}

The main functionality of a PI-HEV is road transportation. This means that the EM strategy should in the first place satisfy the power demand for driving the vehicle. Depending on the available power sources, it will decide how to deliver the requested power in the most economical way. The battery storage capacity offers additional freedom since it can (temporarily) store and retrieve electric energy.

Already many solutions are known to solve the EM problem in HEVs. Especially the on-line optimization strategies, originating from optimal control, show promising results [1]. Here, a global objective function is taken as a starting point to minimize the vehicle's fuel consumption over the entire driving cycle. It turns out to be possible to translate this global optimization problem into an instantaneous minimization procedure, often referred to as fuel equivalent minimization strategy, which can be implemented on-line in the vehicle. In general, these strategies prescribe a desired setpoint for the battery power $P_{s}[\mathrm{~W}]$ by on-line minimization of a fuel function $J[\mathrm{~g} / \mathrm{s}]$ :

$$
\min _{P_{s}} J\left(P_{s}\right)
$$

with $J\left(P_{s}\right)=\dot{m}\left(P_{s}\right)-\lambda P_{s}$, see [9], [10]. The first term $\dot{m}\left(P_{s}\right)[\mathrm{g} / \mathrm{s}]$ describes the instantaneous fuel consumption of the ICE. Note that this function is defined for $P_{s}$ which is closely related to the engine power. After all, if the ICE in an HEV produces more or less power than requested by the driver, the battery with the electric machine take care of the remaining part. This also explains why the second term $\lambda P_{s}$ is present. This term accounts for the energy exchange with the battery and by means of the fuel equivalent factor $\lambda[\mathrm{g} / \mathrm{J}]$, the corresponding fuel use is calculated. Depending on the actual driving situation, $\lambda$ changes and therefore, it needs to be on-line estimated (see e.g. [10] for a solution with a proportional integral controller). Its dimension is explained by the fact that $\lambda$ represents the conversion efficiency for transforming fuel into electric energy.

By connecting the vehicle to the grid, the PI-HEV offers additional freedom to the EM strategy. Rather than changing the aforementioned EM strategy, only minor extensions are necessary to benefit from this extra freedom. In the first place, it is important to realize that the PI-HEV operates either in stationary mode (i.e. plugged-in) or in driving mode, so both modes are never active together. The original fuel equivalent minimization strategy is applied during driving mode. In the stationary mode, logical rules can be used to determine whether it is profitable to exchange energy between the vehicle and the grid. To that end, an energy price is defined for both the grid as well as the vehicle. The price of the grid is defined as $\pi_{g}[€ / J]$ and equals the local price to buy electricity. For the vehicle, the price can be calculated from $\lambda$. Since the EM strategy estimates $\lambda$ on-line, this is a good representation of the fuel costs for producing electric energy with the vehicle. By means of the fuel density $\rho_{f}$ $[\mathrm{g} / \mathrm{l}]$ and the actual fuel price $p_{f}[€ / \mathrm{l}]$, the price $\pi_{f}[€ / J]$ for producing electric energy with fuel is calculated:

$$
\pi_{f}=\frac{p_{f}}{\rho_{f}} \lambda
$$

Note that the unity for the prices $\pi_{f}$ and $\pi_{g}$ can also be defined from a different perspective. Rather than considering pure economic aspects, it is also possible to focus on the environmental impact in terms of $\mathrm{CO}_{2}$ emission. Now the unity for $\pi_{f}$ and $\pi_{g}$ changes from [€/J] into [grams $\left.\mathrm{CO}_{2} / \mathrm{J}\right]$. However, this new situation is not considered in this paper.

To determine wether it is profitable to recharge the battery from the grid, $\pi_{f}$ should be compared to $\pi_{g}$. In the next section, an EM strategy is proposed to solve this optimization problem on-line in the vehicle. In addition, knowledge about future energy prices is valuable information to be included in the decision process. By means of an e-horizon, this information will be taken into account in Section V.

\section{ENERGY MANAGEMENT FOR PLUG-IN VEHICLE}

In the previous section it has been described that a plug-in hybrid vehicle has to deal with two different prices, namely 
$\pi_{f}[€ / J]$ reflecting the costs of electric energy retrieved from the battery when driving with the car and $\pi_{g}[€ / J]$, the costs of electric energy when taken from the electricity grid. In this section an energy management system is proposed that selects the optimal strategy for storing/retrieving electric energy in/from the battery when connected to the grid. This extends the EM strategy described in (1), by means of an additional mode for the plug-in situation, i.e. during stationary mode. First, the idea will be illustrated without taking losses into account.

It is assumed that the electricity grid has dynamic prices, so different prices $\pi_{g i}[€ / J]$ for different time intervals $i$ with length $T_{i}$ [s]. Consider, for convenience, a horizon of $n$ time intervals $i, i=1, n$ and define a positive power flow $P_{i}$ [W] from the grid to the battery. This power is limited by constraints of both the battery $\left[P_{\min }^{b a t}, P_{\text {max }}^{b a t}\right]$ and the power grid connection $\left[P_{\min }^{\text {grid }}, P_{\text {max }}^{\text {grid }}\right]$, so $P_{\min } \leq P_{i} \leq$ $P_{\max }$ with $P_{\text {min }}=\max \left(P_{\text {min }}^{\text {bat }}, P_{\text {min }}^{\text {grid }}\right)<0$ and $P_{\max }=$ $\min \left(P_{\max }^{b a t}, P_{\text {max }}^{\text {grid }}\right)>0$. The energy exchange $E_{i}[\mathrm{~J}]$ in interval $i, E_{i}=P_{i} T_{i}$, has to satisfy the upper limit $E_{\max }$ and lower limit $E_{\min }$ of the energy storage capacity of the battery, which is related to the SOE: State-of-Energy of the battery. The profit of using the energy from the grid instead of retrieving it from the battery in time interval $i$ equals $E_{i} *\left(\pi_{f}-\pi_{g i}\right)[€]$. At the start of the optimization the energy stored in the battery is indicated by $E(0)[\mathrm{J}]$. Now the optimal EM strategy can be formulated. Determine in each time interval $i$ that value of $E_{i}, i=1, n$ which yields maximum profits while satisfying all constraints.

$$
\begin{array}{r}
\max _{E_{i}} \sum_{i=1}^{n} E_{i} *\left(\pi_{f}-\pi_{g i}\right) \\
P_{\text {min }} T_{i} \leq E_{i} \leq P_{\max } T_{i} \quad i=1, n \\
E_{\text {min }} \leq E(0)+\sum_{j=1}^{i} E_{j} \leq E_{\text {max }} \quad i=1, n
\end{array}
$$

This clearly represents a linear programming (LP) optimization problem. As the solution space is bounded, it can be solved fast and easily. The formulation becomes more complex when introducing losses in the AC/DC converter and the battery, as well as battery wear.

Suppose, both losses and wear can be represented by an efficiency coefficient $\eta[-], 0<\eta<1$. Distinguish between the efficiency of storing $\eta^{+}$and retrieving $\eta^{-}$ energy from the battery with $\eta=\eta^{+} \eta^{-}$. Then, there is a difference between the power coming from the grid $P_{i}$ and the power stored in the battery $P_{s i}$ in time interval $i$ with $P_{i}=\max \left(\frac{P_{s i}}{\eta^{+}}, \eta^{-} P_{s i}\right)$. This relation is depicted in Fig. 1.

The constraints on the minimum and maximum power for $P_{s i}$ have to be reformulated as $P_{\text {min }}=\max \left(P_{\text {min }}^{b a t}, \frac{P_{\text {min }}^{g r i d}}{\eta^{-}}\right)<$ 0 and $P_{\max }=\min \left(P_{\max }^{b a t}, \eta^{+} P_{\max }^{\text {grid }}\right)>0$. Now, the energy management strategy can be formulated as follows:

$$
\begin{array}{r}
\max _{P_{s i}} \sum_{i=1}^{n}\left(P_{s i} \pi_{f}-\max \left(\frac{P_{s i}}{\eta^{+}}, \eta^{-} P_{s i}\right) \pi_{g i}\right) T_{i} \\
P_{\text {min }} \leq P_{s i} \leq P_{\max } \quad i=1, n
\end{array}
$$

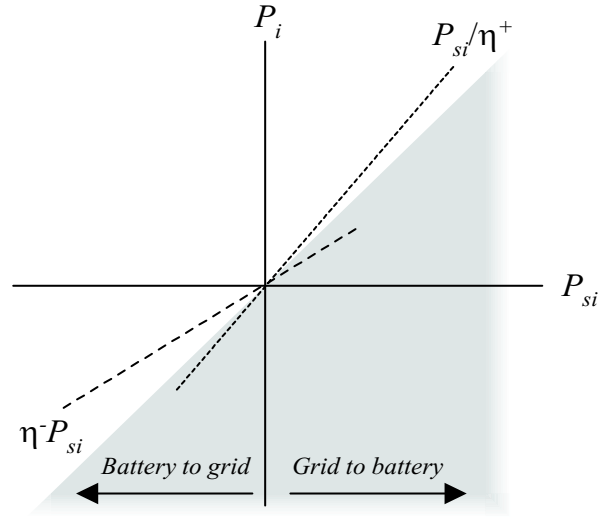

Fig. 1. Model parameters to include losses and wear

$$
E_{\text {min }} \leq E(0)+\sum_{j=1}^{i} P_{s j} T_{j} \leq E_{\max } \quad i=1, n
$$

Again, an LP optimization problem arises. As the solution space is bounded, it can be solved accurately and fast.

The introduction of losses in storing to/retrieving from the battery leads to three different modes of operation depending on the actual price of electricity of the grid $\pi_{g i}$ in time interval $i$ (see Fig. 2):

1) $\pi_{g i}<\eta^{+} \pi_{f}$ : store energy from the grid in the battery

2) $\pi_{g i}>\frac{\pi_{f}}{\eta^{-}}$: retrieve energy from the battery and deliver it to the grid

3) $\eta^{+} \pi_{f} \leq \pi_{g i} \leq \frac{\pi_{f}}{\eta^{-}}$: do nothing.

Owing to these three distinctive modes, the time horizon $(i=1, n)$ of the energy management system falls apart into three separate periods. Each period only consists of consecutive time intervals with equal mode. Within such a period with equal mode the solution is straight forward:

1) Mode 1: if $\pi_{g 1}<\pi_{g 2}<\eta^{+} \pi_{f}$, then the criterion boils down to

$$
\max _{P_{s i}} \sum_{i=1}^{m}\left(\pi_{f}-\frac{\pi_{g i}}{\eta^{+}}\right) P_{s i} T_{i}
$$

In all time intervals during this period with length $m$, the coefficients of $P_{s i} T_{i}$ in the inequality constraint have weighting 1 . So, the weighting in the criterion $\left(\pi_{f}-\frac{\pi_{g i}}{\eta^{+}}\right)$determines which variable will first be increased to maximize the profits. With $m=2$ time intervals the solution will be:

$$
\begin{array}{r}
P_{s 1}=\min \left(P_{\max }, \frac{E_{\max }-E(0)}{T_{1}}\right) \\
P_{s 2}=\min \left(P_{\max }, \max \left(0, \frac{E_{\max }-E(0)-P_{s 1} T_{1}}{T_{2}}\right)\right)
\end{array}
$$

This solution is visualized in Fig. 3. In this example, the energy price from the grid increases after the first time period, so most energy is stored in the battery during the first time period. Nevertheless, the battery 


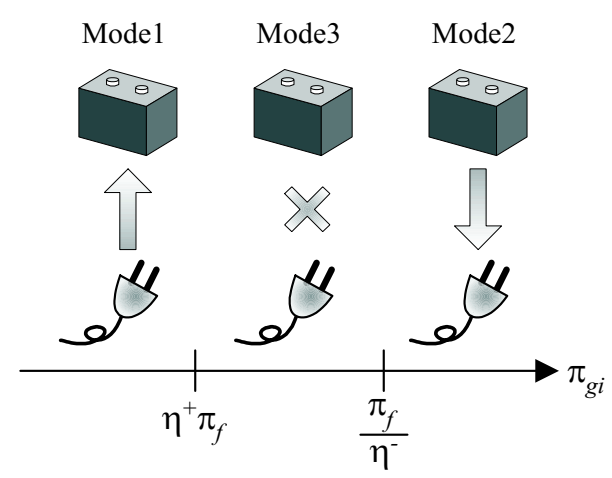

Fig. 2. Plug-in situation covers three modes of operation

will be charged in all time intervals, until the battery is full.

2) Mode 2: in the same way as mode 1, but now the battery is discharged to the grid. If $\pi_{g 1}>\pi_{g 2}>\frac{\pi_{f}}{\eta^{-}}$, then

$$
\begin{array}{r}
P_{s 1}=\max \left(P_{\text {min }}, \frac{E_{\text {min }}-E(0)}{T_{1}}\right) \\
P_{s 2}=\max \left(P_{\text {min }}, \min \left(0, \frac{E_{\text {min }}-E(0)-P_{s 1} T_{1}}{T_{2}}\right)\right)
\end{array}
$$

3) Mode 3: due to losses and battery wear, it is never profitable to exchange energy with the battery, so $P_{s i}=0$.

\section{Remarks}

1) This algorithm not only decides when to retrieve/store electric energy but it also calculates the accurate amount of energy/power that will be exchanged between the battery and the grid in each time period.

2) By considering the energy exchange $E_{i}$ in interval $i$, instead of the power $P_{s i}$, an additional optimization can be made for reducing the wear and/or losses depending on the loading strategy of the battery. When this additional optimization yields a constant value of the power $P_{s i}$ with $P_{s i}=\frac{E_{i}}{T_{i}}$, then there is no difference between optimizing with $E_{i}$ instead of optimizing with $P_{s i}$.

3) Besides a balance in power, the grid must also satisfy constraints on voltages. With the arrival of decentralized power plants (e.g. wind mills, solar panels or micro turbines for both heat and electricity at households) a controllable power source/load has an added value. When properly priced the battery of hybrid or full electric vehicles could be used for these services too.

4) In power systems the ability to supply or consume power peaks has an added value. With large fluctuations in, for example, the production of wind energy or in the consumption, a power source or power load that can quickly adjust its production or consumption when requested is highly valuable. Discussions are going on to create markets for these ancillary services [11].

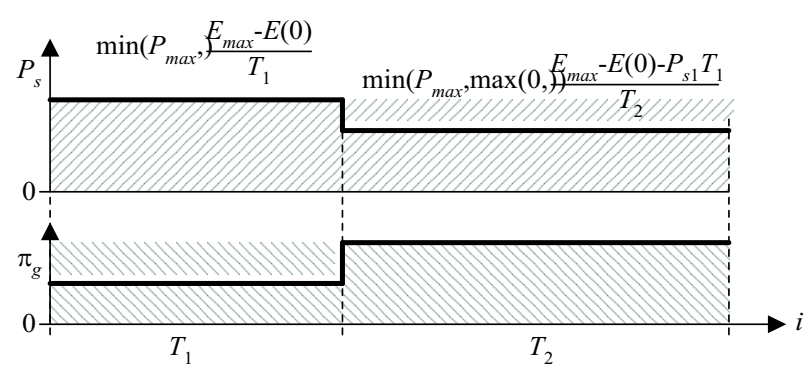

Fig. 3. Example of mode 1 considering two time intervals

Utilities can build their own facilities but the readily available batteries of hybrid vehicles can also supply this service.

\section{Discussion}

In [9] it has been shown that an appropriate value of the fuel equivalent factor $\lambda$ can be on-line estimated. Taking into account a maximum efficiency of the internal combustion engine of about $30-40$ [\%], as well as losses in the generator and taking advantage of energy recovering during regenerative braking, a rough estimate of the ratio between the energy in the fuel and the corresponding electrical energy is about $3: 1$. The energy content of fuel is typically characterized by its lower heating value $h_{f}[\mathrm{~J} / \mathrm{g}]$ and from experiments it is known that $h_{f}$ times the on-line estimated value of $\lambda$ takes values between about 2 and $4[\mathrm{~J} / \mathrm{J}]$, see [9]. From (2) it is possible to calculate the price $\pi_{f}$ and with realistic numbers an estimate for $\pi_{f}$ is about 0.5 [€/kWh].

Large scale electricity production and generation with thermal fossil-fired units can have efficiencies between 30 and $60[\%]$. However, the mix of primary production capacity can also include nuclear and renewables (wind, solar). Together with losses in the transmission and distribution networks it is confusing how to assess an appropriate value for a price $\pi_{g i}$. On the other hand, electricity is already being priced, sometimes depending on fixed periods in a day (day/night). It is to be expected that the price will become more dynamical. For example, at the APX (Amsterdam Power Exchange) the price for electric power is determined in a day ahead market, for time intervals of 1 hour. Considerable changes in the price $\pi_{g i}$ are currently seen during a day or year. In 2007 the lowest price was 0.01 and the highest about 950 [€/MWh], so a ratio of $1: 95.000$ ! Also on a one-day time interval this ratio can change significantly, approximately between 2.5 and 7500. Overnight, price changes between 2 and 3000 are observed. A consumer has to add additional costs such as a transmission fee, metering costs and tax, yielding prices between 0.1 and 0.2 [€/kWh].

So, in almost all situations charging the battery from the grid is the preferred strategy. However, when the price dynamics reach also the consumer, or when connecting to the grid at a company which can buy/sell at APX prices, retrieving electric energy from the battery and supplying it to the grid is an interesting and valid economic-justified 
alternative. But attention has to be paid for the associated costs of a reduced life time of the battery.

Consider, for example, a mid-sized PI-HEV with a battery pack of $9[\mathrm{kWh}$. As stated in [3], this allows for an electric driving range of approximately $30[\mathrm{~km}]$. In case the vehicle is daily used for commuter traffic, full electric drive is possible as long as the single distance covered is less than $30[\mathrm{~km}]$. Now suppose that the price for fuel and electricity equals $\pi_{f}=0.5[€ / \mathrm{kWh}]$ and $\pi_{g}=0.15[€ / \mathrm{kWh}]$, respectively. Then the energy costs can be reduced up to $\left(1-\pi_{g} / \pi_{f}\right) \times 100=70[\%]$, without considering losses and battery wear. This potential benefit will be lower due to a limited energy efficiency, but also due to trips larger than $30[\mathrm{~km}]$. On the other hand, energy losses up to 70 [\%] are allowed in the battery and the $\mathrm{AC} / \mathrm{DC}$ converter (i.e. $\left.\eta^{+}>0.3[-]\right)$, to charge the battery from the grid and still gain profits. Hence, it is reasonable to assume that electricity from the grid is preferred to charge the batteries.

\section{ELECTRONIC HORIZON}

The electronic horizon (e-horizon) is a useful method to further optimize the performance of the EM system. Historically, the e-horizon concept was introduced to inform the EM system about upcoming driving situations. Typically, information about the future driving cycle is evaluated to predict the fuel equivalent factor $\lambda$ and adapt the EM strategy [12]. Alternatively, the e-horizon can also be implemented by describing a preferred reference trajectory for the battery SOE (State-of-Energy) [13]. Here it will be shown that this latter concept will be useful in stationary applications too (when the vehicle is connected to the grid) to improve the performance and robustness of the EM system.

Important for the robustness of the EM system is how to deal with constraints on the battery storage capacity. In all HEVs, the storage capacity is ultimately bounded and by anticipating on upcoming events, the available capacity is further exploited. Think for example of a trip through the mountains where energy from regenerative braking is frequently available. Since the e-horizon prescribes a desired SOE-reference trajectory, it can guarantee that the battery is never full prior to a braking period and the free regenerative braking energy can always be stored, see [13].

With respect to a PI-HEV, this latter concept of prescribing a reference trajectory for the SOE can be easily extended to take into account the energy exchange with the grid. Suppose that the energy price $\pi_{g}$ is low enough such that the vehicle will be charged overnight from the grid. A preferred SOE reference trajectory is characterized by a full battery when the vehicle starts driving $(\operatorname{SOE}(0)=$ $\left.S O E_{\max }=100[\%]\right)$ and needs to end the trip with an empty battery $\left(S O E\left(T_{\text {end }}\right)=S O E_{\min }\right)$. During driving, the e-horizon schedules the energy from the battery according to information about the expected driving cycle. All these requirements are easily incorporated in the algorithm as described in [13].

In Section III, three modes of operation are identified when the vehicle is connected to the grid, i.e. in stationary mode. In
TABLE I

ACTIONS TAKEN BY THE E-HORIZON

\begin{tabular}{lll}
$\begin{array}{l}\text { Stationary situation } \\
\text { (Connected to grid) }\end{array}$ & $\begin{array}{l}\text { Driving situation } \\
\text { (Disconnected from grid) }\end{array}$ \\
\hline $\begin{array}{l}\text { Mode 1: } \\
\text { Battery charging from grid }\end{array}$ & $\Leftrightarrow$ & Discharge battery along journey \\
$\begin{array}{l}\text { Mode 2: } \\
\text { Battery discharging to grid } \\
\text { Mode 3: }\end{array}$ & $\Leftrightarrow$ & Charge battery along journey \\
No net energy exchange & $\Leftrightarrow$ & Keep SOE constant \\
\hline
\end{tabular}

accordance with these three situations, Table I summarizes the actions to be taken by the e-horizon. It appears that, also for driving, the e-horizon discriminates between three modes. These three modes are characterized by an initial value and a final value for the SOE reference trajectory during driving. The algorithm from [13] will be used to calculate the SOE trajectory in between. Since the algorithm includes information about the expected diving cycle, it will be able to anticipate on the driving style of the driver.

During a connection overnight it could happen that the battery is both charged and discharged depending on the actual prices $\pi_{g i}$ of electricity in each time interval. Consequently, only the net charging/discharging is denoted in Table I, whereas the battery can act as a storage buffer to the grid.

With respect to the dynamical energy market, the e-horizon also offers an important contribution for the EM system when the vehicle is connected to the grid. Traditionally, the price for electric energy was fixed overnight and the e-horizon should concentrate on how to optimize the energy exchange with the grid, such that losses and wear of the battery are prevented. If the vehicle, for example, remains connected to the grid during the entire night, the e-horizon can schedule the energy from/to the battery without requiring high power peaks to prevent losses and battery wear.

In a dynamical energy market, however, the energy prices are not fixed. Since market prices change according to the law of supply and demand, it is reasonable to believe that energy prices change periodically with the time of the day. In that case, historical data or the prices determined and published by energy exchange organizations, such as APX, can be used to predict upcoming energy prices when the vehicle is connected to the grid. If, for example, the energy price decreases during the night, battery charging should preferably be done when minimum prices are reached, so at moments that are economically most attractive.

\section{CONCLUSIONS AND OUTLOOK}

The advanced power net configuration of a PI-HEV allows vehicle propulsion from various power sources. In a dynamical energy market, prices for energy change over time and the EM system has to decide how and when to use the available power sources. The EM system proposed in this paper extends the fuel equivalent minimization strategy known from HEVs and takes into account the plug-in situation and executes an on-line optimization algorithm. 
The EM system utilizes knowledge of prices for energy taken by the PI-HEV from the grid, and of the price of electric energy stored in its battery. Then, the proposed energy management system determines accurately a charging/discharging strategy which yields maximum profits. The larger the differences in prices of the electricity grid among the time intervals of a future time horizon, the larger profits can be achieved. When customers keep their PI-HEV available for the electricity net responsible party, the ability to store/retrieve quickly electric power has its own economic rewards. With an expected liberalization of the energy markets and an increasing penetration of less-predictable renewables, the value of readily available power (either production or consumption) has an attractive economic potential.

The PI-HEV will benefit from an e-horizon for both driving and stationary application. During the driving mode, the e-horizon will be helpful to schedule the energy in the battery such that the vehicle utilizes the energy source which is most profitable. Conversely, the stationary situation benefits from the e-horizon by defining an optimal charging/discharging strategy for the battery which takes into account the predicted price for electric energy in the near future. Characteristics which lead to losses or battery wear can be easily taken into account and further optimize the energy exchange between the vehicle and the grid.

\section{REFERENCES}

[1] A. Sciarretta and L. Guzzella, "Control of hybrid electric vehicles," IEEE Control Systems Magazine, vol. 27, no. 2, pp. 60-70, April 2007.

[2] C. Chan, "The state of the art of electric, hybrid, and fuel cell vehicles," Proceedings of the IEEE, vol. 95, no. 4, pp. 704-718, April 2007.

[3] W. Jones, "Take this car and plug it," IEEE Spectrum, vol. 42, no. 7, pp. 10-13, July 2005.

[4] R. Winkel, R. van Mieghem, D. Santini, M. Duvall, V. Conte, M. Alaküla, F. Badin, R. Bleis, A. Brouwer, and P. Debal, "Global prospects of plug-in hybrids," in Proc. of the EVS 22 - Electric Vehicle Symposium, Yokohama, Japan, October 2006.

[5] C. Mohrdieck, A. Truckenbrodt, and K. Noreikat, "Plug-In Hybrids - Promise, Hype or the Solution?" in Proc. of the EVS 23 - Electric Vehicle Symposium, Anaheim, California, December 2007.

[6] A. Frank, "Battery needs for plug-in hybrid vehicles," in Proc. of the EVS 20 - Electric Vehicle Symposium, Long Beach, California, November 2003.

[7] D. Karbowski, A. Rousseau, S. Pagerit, and P. Sharer, "Plug-in vehicle control strategy: From global optimization to real-time application," in Proc. of the EVS 22 - Electric Vehicle Symposium, Yokohama, Japan, October 2006.

[8] J. Hafner and J. Tomlin, "System and method for minimizing energy consumption in hybrid vehicles," U.S. Patent US 7,013,205 B1, 2006.

[9] J. Kessels, M. Koot, B. de Jager, P. van den Bosch, N. Aneke, and D. Kok, "Energy management for the electric powernet in vehicles with a conventional drivetrain," IEEE Trans. on Control Systems Technology, vol. 15, no. 3, pp. 494-505, May 2007.

[10] M. Koot, J. Kessels, B. de Jager, W. Heemels, P. van den Bosch, and M. Steinbuch, "Energy management strategies for vehicular electric power systems," IEEE Trans. on Vehicular Technology, vol. 54, no. 3, pp. 771-782, May 2005.

[11] A. Jokić, "Price-based optimal control of electrical power systems," Ph.D. dissertation, Department of Electrical Engineering, Technische Universiteit Eindhoven, The Netherlands, 2007.

[12] C. Musardo, G. Rizzoni, and B. Staccia, "A-ECMS: an adaptive algorithm for hybrid electric vehicle energy management," in Proc. of the joint 44th IEEE Conf. on Decision and Control \& European Control Conference, Seville, Spain, December 2005, pp. 1816-1823.

[13] J. Kessels and P. van den Bosch, "Electronic horizon: Energy management using telematics information," in Proc. of the IEEE Vehicle Power and Propulsion Conference, Arlington, Texas, September 2007. 